\title{
Infectivity of a rickettsia isolated from coho salmon Oncorhynchus kisutch
}

\author{
L. H. Garcés ${ }^{1}$, J. J. Larenas ${ }^{1}$, P. A. Smith ${ }^{1}$, S. Sandino ${ }^{2}$, C. N. Lannan ${ }^{3}$, J. L. Fryer ${ }^{4, *}$ \\ ${ }^{1}$ Veterinary Sciences Faculty, University of Chile, Santiago, Chile \\ ${ }^{2}$ Association of Chilean Salmon Farmers, Puerto Montt, Chile \\ ${ }^{3}$ Laboratory for Fish Disease Research, Department of Microbiology, Oregon State University, Hatfield Marine Science Center, \\ Newport, Oregon 97365-5296, USA \\ ${ }^{4}$ Department of Microbiology, Oregon State University, Nash Hall 220, Corvallis, Oregon 97331-3804, USA
}

\begin{abstract}
Two species of salmonids were tested for their susceptibility to infection by a rickettsia isolated in cell culture from diseased coho salmon Oncorhynchus kisutch in Chile. Mortality approached $100 \%$ in coho and Atlantic salmon Salmo salar injected with 10 -fold dilutions of cell culture medium containing the rickettsia. Typical disease signs were present in the coho salmon but were not observed in inoculated Atlantic salmon. However, the rickettsia was recovered in pure culture from moribund fish in each of the injected groups of either species. The rickettsia was thereby demonstrated to be pathogenic and the cause of the ongoing epizootic affecting salmonids cultured in Chile. Horizontal transmission was not demonstrated in a group of uninoculated coho salmon held in the same tank with experimentally infected fish.
\end{abstract}

\section{INTRODUCTION}

Salmonid aquaculture is a rapidly growing industry in the sheltered coastal waters of southern Chile; however, coho salmon Oncorhynchus kisutch reared in seawater netpens in this area have experienced an ongoing epizootic first documented by Bravo \& Campos (1989). The losses described were extensive, and, at certain sites, mortality as great as $90 \%$ was attributed to this disease. Although the epizootic was initially thought to be confined to coho salmon, other salmonid species have subsequently been affected. Mortality has occurred in Atlantic salmon Salmo salar and chinook salmon Oncorhynchus tshawytscha cultured in the area, and rainbow trout Oncorhynchus mykiss have also experienced losses to this disease.

Moribund coho salmon collect at the corners of the netpens when an epizootic is in progress. These fish are lethargic and dark in color. Hematocrits are low (ca $27 \%$ ), the gills are pale, the kidney is swollen and the spleen enlarged. Occasionally, the liver has a gray, mottled appearance (Bravo \& Campos 1989). Severe necrosis and inflammation in the liver, spleen, intestine, and hematopoietic tissue of the kidney can be

- Author for correspondence seen. Mild lesions in pancreatic, cardiac, and ovarian tissues may also be present (Cvitanich et al. 1990).

In 1989, as part of an extensive effort to determine the cause of the continuing epizootic, kidney tissue from moribund coho salmon was inoculated onto a chinook salmon embryo cell line (CHSE-214) (Lannan et al. 1984), and a previously undescribed rickettsia was isolated in these cell cultures. The isolation and in vitro characteristics of this agent were reported by Fryer et al. (1990). The rickettsia is pleomorphic, although the morphology is predominantly coccoid, and the diameter of individual organisms ranges between 0.5 and $1.5 \mu \mathrm{m}$. It is an obligately intracellular parasite and produces cytopathic effect (CPE) in cultured fish cells, where it replicates within membrane-bound cytoplasmic inclusions. It does not replicate on any of the commonly-used bacteriological media. The optimum temperature range for replication is 15 to $18^{\circ} \mathrm{C}$. Replication is inhibited by the presence of streptomycin, gentamicin, and tetracycline, but not by penicillin. Based on morphology and the site of replication, the rickettsia was tentatively placed in the family Rickettsiaceae and the tribe Ehrlichieae.

This report documents the quantitative experimental infection of coho and Atlantic salmon with the rickettsia isolated from coho salmon in 1989. It details the 
production of the disease with accompanying mortality, and the recovery of the etiologic agent from the experimentally infected fish.

\section{MATERIALS AND METHODS}

Fish. The infectivity study was conducted using juvenile coho and Atlantic salmon, weighing ca $10 \mathrm{~g}$ each. These fish were reared from eggs imported from the Pacific Northwest of the USA and had been held only in fresh water. Groups of 60 coho salmon and groups of ca 40 Atlantic salmon were transferred to $600 \mathrm{l}$, circular, fiberglass tanks supplied with flowthrough, specific-pathogen-free, filtered spring water. The inflow water was passed through a Jacuzzi Model MSM-24 sand filtration system (Jacuzzi, Inc., Iittle Rock, AR, USA) and delivered to the tanks at a flow rate of ca $61 \mathrm{~min}^{-1}$. The water temperature ranged from 9 to $12^{\circ} \mathrm{C}$ throughout the experiment, with a mean daily water temperature of $10.5^{\circ} \mathrm{C}$. Effluent water from the tanks was treated with sodium hypochlorite in sufficient concentration to kill pathogens present.

Rickettsial isolate. The rickettsia used for inoculation was isolated from a moribund 2-yr-old coho salmon, ca $40 \mathrm{~cm}$ in length and weighing ca $1 \mathrm{~kg}$. The salmon displayed typical signs of the disease and was collected from a seawater netpen in southern Chile where a natural epizootic was in progress. Kidney tissue was aseptically removed from the fish and inoculated directly into $25 \mathrm{~cm}^{2}$ tissue culture flasks (Corning Glass Works, Corning, NY, USA) containing a monolayer of CHSE-214 cells in antibiotic-free Eagle's Minimum Essential Medium with Earle's salts (Automod, Sigma Chemical Co., St. Louis, MO, USA), supplemented with $10 \%$ fetal bovine serum (Hyclone Laboratories, Inc. Logan, UT, USA) (MEM-10). The cultures were kept at $4{ }^{\circ} \mathrm{C}$ for transport to the Oregon State University Hatfield Marine Science Center, Laboratory for Fish Disease Research, Newport, Oregon, USA. Upon arrival, they were incubated at $15^{\circ} \mathrm{C}$ and observed for the appearance of CPE.

In the primary isolation, clusters of rounded cells (Fig. 1) appeared in the monolayer after $10 \mathrm{~d}$ incubation at $15^{\circ} \mathrm{C}$. With time, the clusters increased in size and number until the entire cell sheet was affected Ultimately, all the CHSE-214 cells detached from the flask and floated free in the medium. When CPE in the original flasks became extensive, an aliquot of spent culture medium was transferred to fresh monolayers of CHSE-214 cells, and these cultures were incubated and observed. In subsequent passages, CPE appeared in 5 to $6 \mathrm{~d}$ and was complete in ca $14 \mathrm{~d}$. Within $48 \mathrm{~h}$ of inoculation, rickettsiae could be observed within cytoplasmic vacuoles or inclusions in fixed and stained CHSE-214 cells (Fig. 2).

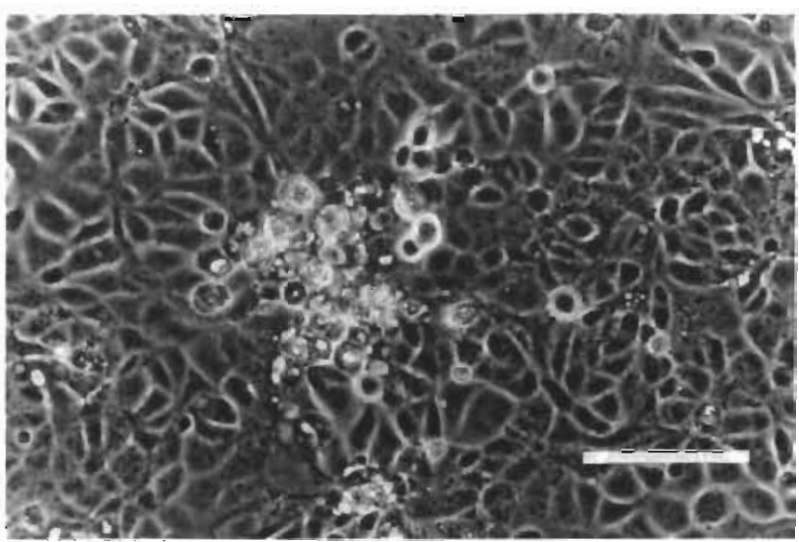

Fig. 1. Cytopathic effect produced by the rickettsia in CHSE214 cells. Phase contrast. Bar $=100 \mu \mathrm{m}$

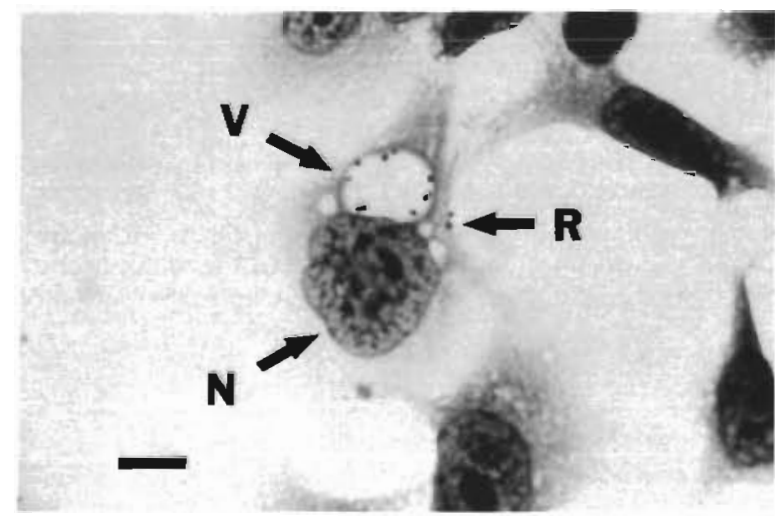

Fig. 2. Rickettsia within a cytoplasmic vacuole (V) in a CHSE214 cell $2 \mathrm{~d}$ post inoculation. Two rickettsiae (R) are also attached to the outer membrane of the host cell. $N=$ host cell nucleus. May Greenwald-Giemsa stain. Bar $=10 \mu \mathrm{m}$

For use in the challenge experiments, medium was harvested from a culture in which the CPE was near completion (ca $12 \mathrm{~d}$ ). A series of 10-fold dilutions of the spent medium, containing the rickettsia, was made up in MEM-10 without antibiotics. These dilutions of the rickettsial suspension were used to inoculate the fish.

The infectivity titer of the inoculum was determined by endpoint dilution assay $\left(\mathrm{TCID}_{50}\right.$ ) using $\mathrm{CHSE}-214$ cells in a 96 -well tissue culture plate (Corning). The dilution endpoints were calculated by the method of Reed \& Muench (1938)

Challenge. The coho salmon were challenged by injection with 3 concentrations of the rickettsial suspension. They were injected intraperitoneally (IP) with $10^{5.3}, 10^{4.3}$, or $10^{3.3}$ TCID $_{50}$ in $0.05 \mathrm{ml}$. Sixty uninoculated control fish were held under the same conditions as the experimental specimens. Mortalities were collected and recorded daily for $42 \mathrm{~d}$. The Atlantic salmon were injected IP with $10^{5.3}$ or $10^{4.3}$ TCID $_{50}$ fish $^{-1}$ Atlan- 
tic salmon mortalities were collected and recorded for $39 \mathrm{~d}$.

Recovery of etiologic agent. Kidney tissue from moribund fish in each experimental group was inoculated onto monolayers of CHSE-214 cells in antibioticfree culture medium. The cultures were incubated at $15^{\circ} \mathrm{C}$ and observed for the appearance of CPE. An aliquot of spent medium from flasks showing CPE was transferred to fresh CHSE-214 cells, and these cells were further incubated and observed. In addition, smears were made from the spent medium collected from positive cultures. The smears were air-dried, fixed in absolute methanol, Giemsa-stained, and microscopically examined to verify the presence of the rickettsia.

Horizontal transmission. Twenty uninoculated coho salmon were held in a cage in the same tank with coho salmon injected with the highest concentration of the rickettsia $\left(10^{5.3}\right.$ TCID $_{50}$ fish $\left.^{-1}\right)$. The fish were observed daily for mortalities, and, on the final day of the experiment (Day 42), Giemsa-stained blood and kidney tissue smears from 10 fish were examined microscopically to determine if the rickettsia was present. In addition, kidney tissue from one fish was inoculated onto CHSE214 cells. This culture was incubated and observed in the same way as those already described.

\section{RESULTS}

The coho salmon disease, as described by Bravo \& Campos (1989), was produced in all of the groups of coho salmon injected with the rickettsia, but not in uninoculated fish in the horizontal transmission experiments or in any of the uninoculated controls. As the disease progressed, infected coho salmon became lethargic, anorectic, and dark in color. These fish congregated at the surface of the water near the edge of the tank. Many became disoriented, whirling or swimming into the walls of the tank.

Moribund fish were anemic. The gills and liver were pale, the spleen enlarged, and both the kidney and spleen were congested. Many fish had hemorrhages at the base of the fins, and there were petechial hemorrhages on the pyloric caeca. The digestive tract was fluid-filled but devoid of food. In a few fish, the abdomen was distended and filled with ascities.

It was not possible to calculate an $\mathrm{LD}_{50}$ value for these fish, as mortality approached $100 \%$ in all of the challenged groups; however, the mortality followed a clear dose-response pattern. In Group 1, experimental specimens were inoculated with $10^{5.3} \mathrm{TCID}_{50} \mathrm{fish}^{-1}$; mortality began on Day 16, peaked on Day 18, and reached $100 \%$ by Day 33 (Fig. 3a). Group 2 was inoculated with $10^{4.3}$ TCID $_{50}$ fish $^{-1}$; mortality began on Day 18 , peaked on Day 21 , and ultimately reached $98 \%$ by a

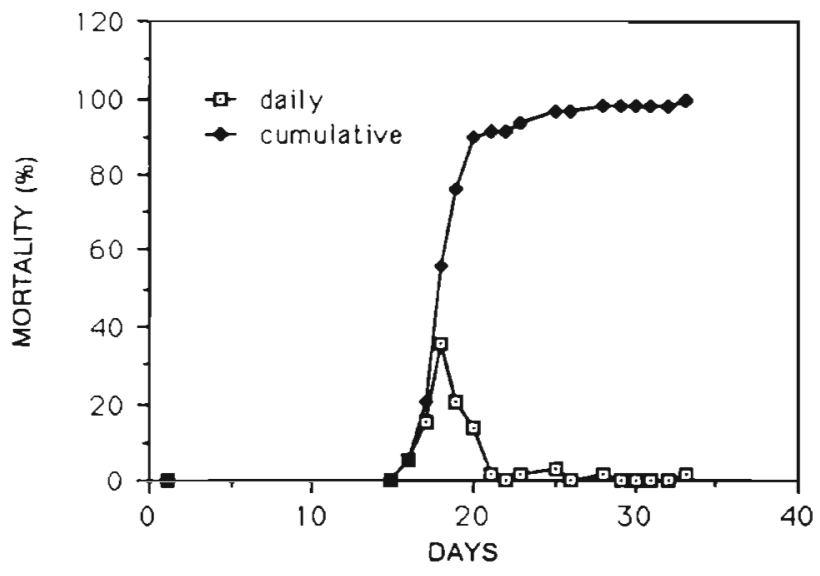

b

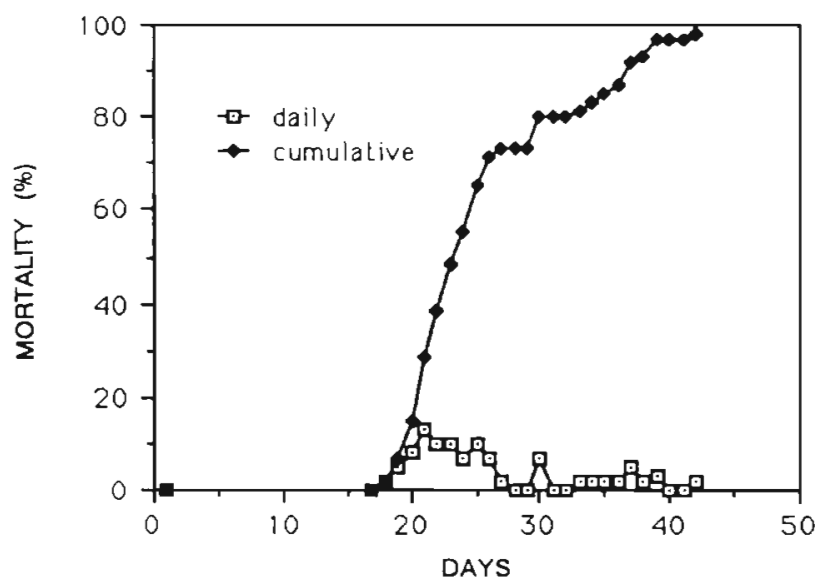

C

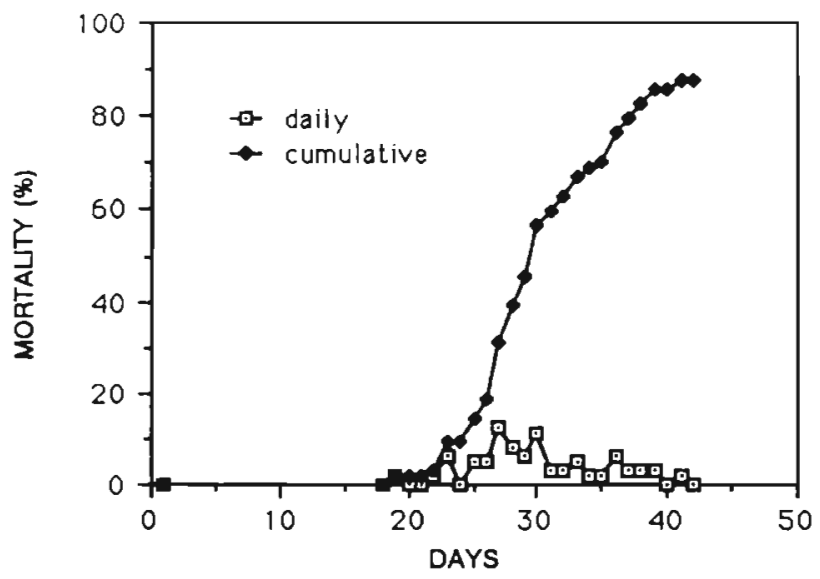

Fig. 3. Oncorhynchus kisutch. Daily and cumulative mortality $(\%)$ of coho salmon inoculated with the coho salmon rickettsia. (a) Inoculated with $10^{5.3} \mathrm{TCID}_{50} \mathrm{fish}^{-1}$; (b) inoculated with $10^{4.3} \mathrm{TCID}_{50}$ fish $^{-1}$; (c) inoculated with $10^{3.3} \mathrm{TCID}_{50}$ fish $^{-1}$

Day 42 (Fig. 3b). In the third group, inoculated with $10^{3.3}$ TCID $_{50}$ fish $^{-1}$, mortality began on Day 19 and peaked on Day 27 (Fig. 3c). Mortality in this group continued at a low rate until the experiment was termi- 
nated on Day 42 , but had reached $88 \%$ at that time The mean day to death was Day 18 for Group 1, Day 24 for Group 2, and Day 29 for Group 3.

The rickettsia was isolated in pure culture from moribund coho salmon in each injected group, completing Koch's postulates. No rickettsiae were recovered in the cell culture inoculated with tissue from the horizontal transmission test. No fish died in this group, there was no evidence of disease among them, and no rickettsiae were observed in blood and kidney smears from these fish. However, tissue from only one fish was screened in cell culture to determine if the rickettsia was present.

Mortality levels produced in Atlantic salmon injected with the rickettsia were similar to those recorded in the challenged coho salmon Death began on Day 14 and reached $100 \%$ in Atlantic salmon inoculated with $10^{5.3}$ TCID $_{50}$ fish $^{-1}$. Mortality in the second group $\left(10^{4.3}\right.$ TCID $_{50}$ fish $^{-1}$ ) began on Day 18 and had reached $93 \%$ when the experiment was terminated on Day 39 (Fig. 4). No other disease signs were apparent, but the rickettsia was isolated in pure culture from moribund fish.

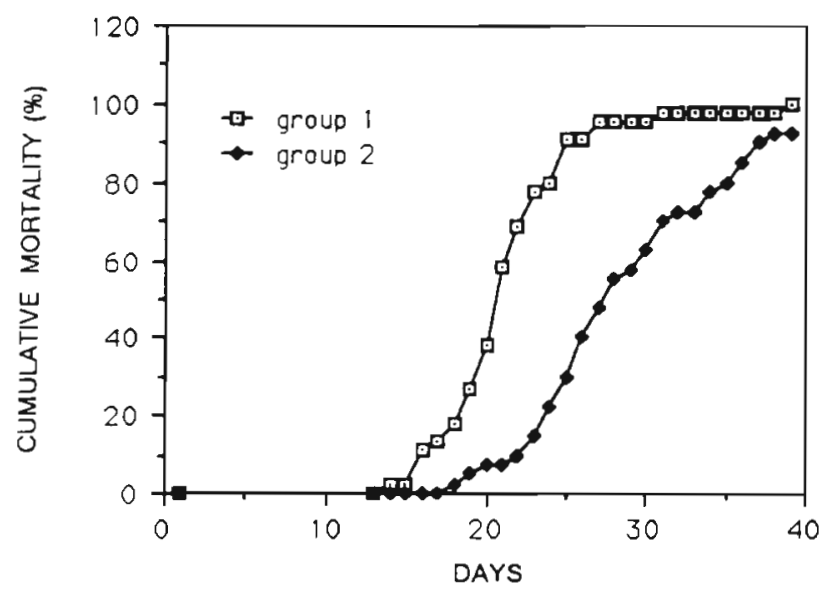

Fig. 4. Salmo salar. Cumulative mortality (\%) of Atlantic salmon inoculated with the coho salmon rickettsia. Group 1 was inoculated with $10^{5.3} \mathrm{TCID}_{50} \mathrm{fish}^{-1}$. Group 2 was inoculated with $10^{4.3}$ TCID $_{50}$ fish $^{-1}$

\section{DISCUSSION}

The infectivity experiments reported here demonstrate that the rickettsia isolated from coho salmon in 1989 is the cause of the coho salmon disease that has produced major losses among cultured salmonids in Chile. Mortality ranging from 88 to $100 \%$ was observed in groups of coho and Atlantic salmon injected with this organism, and it is apparent that the rickettsia is virulent in both of these salmonid species.

Recovery of the rickettsia from experimental fish posed a difficult problem, as conditions for necropsy were less than optimal, and the agent is susceptible to the antibiotics commonly employed in cell culture. Nevertheless, the rickettsia was isolated in pure culture from moribund fish in each injected group, completing Koch's postulates.

Diseases with a confirmed rickettsial etiology have not previously been reported in fish. We initially thought that this was the first rickettsia isolated from a fish species, but we have since become aware of the observation by Ozel \& Schwanz-Pfitzner (1975) of a rickettsia-like agent in cultured fish cells inoculated with tissue from rainbow trout infected with Egtved virus (VHSV). The rainbow trout, collected from a freshwater system, were apparently host to both VHSV and an agent with morphological characteristics similar to the rickettsiae. Although mortality was noted in the trout population, the cause was unclear. No studies were conducted, beyond microscopic observation, to determine if the agent seen in the cell cultures was, in fact, a rickettsia or if it was capable of producing disease in fish. Unfortunately, the agent observed was not maintained and is no longer available for study or comparison (I. Schwanz-Pfitzner, Robert Koch-Institut, Berlin, pers. comm. 1990).

In the present study, transmission of the coho salmon rickettsia was accomplished by injection, leaving undefined the method of natural transmission (horizontal, vertical, via a vector) of this agent. Horizontal transmission did not occur between injected and noninjected fish held in the same tank. However, this work was conducted in flowing, fresh water, creating an environment different from the seawater netpens where the coho salmon disease has been observed. Other researchers have stated that fish died following injection of this organism (Cvitanich et al. 1990), and that horizontal transmission occurred between injected and noninjected individuals, but no quantification or details of the work were provided.

The rickettsia isolated from coho salmon is, clearly, a serious pathogen of potential concern to the entire salmonid culture industry, and the development of methods and reagents for detection and control of this organism are of high priority. There exists a particular need for methodology to detect the presence of the rickettsia in infected fish and fish products to prevent the transfer of the agent from areas where it is enzootic to areas where it does not presently occur.

Two methods are currently available for detection of the coho salmon rickettsia: microscopic examination of Giemsa-stained material or isolation of the agent in cell culture. However, detection of the rickettsia in Giemsastained tissues is not easily accomplished when low numbers are present, and isolation of the organism from infected fish is made difficult by the requirement of conducting these assays in cells cultured in antibiotic- 
free medium. Therefore, assays that are sensitive and simple to perform are needed.

In addition to the need for improved methods of detection of the rickettsia, it is imperative that efforts be made to control the spread of the disease. In order to accomplish this, work must be undertaken to identify the source of the infection in the seawater environment and to elucidate the mode of transmission of the agent under natural conditions. Of equal importance is the determination of efficacious therapeutic agents for treatment of the disease and the development of immunogenic preparations (vaccines) for preventing the disease.

Acknowledgements. We thank Ms Helene Vega for her invaluable aid with the collection of data for this study. This work was sponsored by the Association of Chilean Salmon Farmers (Salmolab S.A. and Marine Harvest Chile), by Oregon Sea Grant with funds from the National Oceanographic and Atmospheric Administration Office of Sea Grant, Department of Commerce, under grant no. NA89AA-D-SG108, and by the Veterinary Sciences Faculty of the University of Chile.

Responsible Subject Editor; $T$ Evelyn, Nanaimo, B. C., Canada
The report is Oregon Agricultural Experiment Station Technical Paper No. 9423

\section{LITERATURE CITED}

Bravo, S., Campos, M. (1989). Coho salmon syndrome in Chile. FHS/AFS Newsletter 17 (3): 3

Cvitanich, J., Garate, O. Smith, C. E. (1990). Etiological agent in a Chilean coho disease isolated and confirmed by Koch's postulates. FHS/AFS Newsletter 18 (1): 1-2

Fryer, J. L., Lannan, C. N., Garcés, L. H., Larenas, J. J., Smith, P. A. (1990). Isolation of a rickettsiales-like organism from diseased coho salmon (Oncorhynchus kisutch) in Chile. Fish Pathol. 25: 107-114

Lannan, C. N., Winton, J. R, Fryer, J. L. (1984). Fish cell lines establishment and characterization of nine cell lines from salmonids. In Vitro 20: 671-676

Ozel, M., Schwanz-Pfitzner, I. (1975). Vergleichende elektronenmikroskopische Untersuchungen an Rhabdoviren pflanzlicher und tierischer Herkunft: III. EgtvedVirus (VHS) der Regenbogenforelle (Salmo garidneri) und Rickettsienähnliche Organismen. Zbl. Bakt. Hyg., 1. Abt. Orig. 230: 1-14

Reed, L. J., Muench, H. (1938). A simple method of estimating fifty percent endpoints. Am. J. Hyg. 27: 493-497

Manuscript first received: January 5, 1991

Revised version accepted: April 18, 1991 